\title{
Enxerto e transferência de nervo para melhora da flexão do cotovelo em crianças com paralisia obstétrica. Uma revisão sistemática*
}

\section{Nerve Graft and Nerve Transfer for Improving Elbow Flexion in Children with Obstetric Palsy. A Systematic Review}

\author{
Eduardo Velásquez-Girón ${ }^{10}$ James A. Zapata-Copete ${ }^{2}$ ] \\ ${ }^{1}$ Seção de Cirurgia Ortopédica de Mão, Departamento de Cirurgia, \\ Endereço para correspondência Eduardo Velásquez Girón, MD, MSc, \\ Universidad del Valle, Cali, Colômbia \\ 2 Departamento de Cirurgia, Seção de Cirurgia Plástica, Universidad \\ Department of Surgery, Orthopedic Hand Surgery Section, \\ Universidad del Valle, Street 4b \# 36-00, Cali, Colombia \\ del Valle, Cali, Colômbia \\ (e-mail: eduardo.velasquez@correounivalle.edu.co).
}

Rev Bras Ortop 2021;56(6):705-710.

\section{Resumo}

Palavras-chave

- paralisia do plexo braquial neonatal

- transferência de nervo

- enxertos alogênicos
A paralisia obstétrica do plexo braquial é uma lesão bastante comum em neonatos, sendo causada pela tração do plexo braquial durante o trabalho de parto. A presente revisão sistemática tem como objetivo explorar o uso de enxertos e transferências de nervo como procedimentos para melhora da flexão do cotovelo em crianças com paralisia obstétrica. A presente revisão sistemática seguiu as diretrizes Preferred Reporting Items for Systematic Reviews and Meta-Analyses (PRISMA, na sigla em inglês) e foi baseada em pesquisa nos bancos de dados MEDLINE, EMBASE, LILACS, The Cochrane Central Register of Controlled Trials, Web of Science, Wholis e SCOPUS. De acordo com os critérios pré-determinados, os artigos incluídos eram ensaios clínicos, quase-experimentos, e estudos de coortes sobre enxertos e transferências de nervos em crianças (de até 3 anos de idade) com diagnóstico de paralisia obstétrica. A ferramenta de avaliação Risk of Bias in Non-Randomized Studies of Interventions foi usada em estudos não randomizados. Sete estudos utilizaram os dois procedimentos; três deles compararam os procedimentos de enxerto e transferência de nervo, enquanto os outros quatro os combinaram como método reconstrutivo em crianças com paralisia obstétrica. Segundo o sistema de classificação do Medical Research Council, os dois métodos melhoraram a flexão do cotovelo das crianças de maneira similar. De modo geral, nossos resultados mostraram que o enxerto de nervo e a transferência de nervo são opções igualmente boas para a reconstrução nervosa em casos de paralisia obstétrica. Mais estudos sobre as técnicas de reconstrução nervosa
Estudo desenvolvido na Seção de Cirurgia Ortopédica de Mão, Departamento de Cirurgia, Universidad del Valle, Cali, Colômbia. recebido

31 de Agosto de 2020

aceito

17 de Dezembro de 2020

Publicado on-line

Agosto 13, 2021
DOI https://doi.org/

10.1055/s-0041-1729586. ISSN 0102-3616.
(C) 2021. Sociedade Brasileira de Ortopedia e Traumatologia. All rights reserved.

This is an open access article published by Thieme under the terms of the Creative Commons Attribution-NonDerivative-NonCommercial-License, permitting copying and reproduction so long as the original work is given appropriate credit. Contents may not be used for commercial purposes, or adapted, remixed, transformed or built upon. (https://creativecommons.org/ licenses/by-nc-nd/4.0/)

Thieme Revinter Publicações Ltda., Rua do Matoso 170, Rio de Janeiro, RJ, CEP 20270-135, Brazil 
Abstract

\author{
Keywords \\ - obstetrical brachial \\ plexus palsy \\ - nerve transfer \\ - allogenic grafts
}

na paralisia obstétrica devem ser realizados, de preferência ensaios clínicos randomizados, para validação dos resultados dessa revisão sistemática.

Obstetric brachial plexus palsy is a rather common injury in newborns, caused by traction to the brachial plexus during labor. In this context, with the present systematic review, we aimed to explore the use of nerve graft and nerve transfer as procedures to improve elbow flexion in children with obstetric palsy. For the present review, we followed the Preferred Reporting Items for Systematic Reviews and Meta-Analyses (PRISMA) guidelines. We searched the MEDLINE, EMBASE, LILACS, The Cochrane Central Register of Controlled Trials, Web of Science, Wholis and SCOPUS databases. Predetermined criteria defined the following requirements for inclusion of a study: Clinical trials, quasi-experiments, and cohort studies that performed nerve graft and nerve transfer in children ( $\leq 3$ years old) with diagnosis of obstetric palsy. The risk of bias in nonrandomized studies of interventions assessment tool was used for nonrandomized studies. Out of seven studies that used both procedures, three of them compared the procedures of nerve graft with nerve transfer, and the other four combined them as a reconstructive method for children with obstetric palsy. According to the Medical Research Council grading system, both methods improved equally elbow flexion in the children. Overall, our results showed that both techniques of nerve graft and nerve transfer are equally good options for nerve reconstruction in cases of obstetric palsy. More studies approaching nerve reconstruction techniques in obstetric palsy should be made, preferably randomized clinical trials, to validate the results of the present systematic review.

\section{Introdução}

A lesão obstétrica do plexo braquial (LOPB) ou paralisia obstétrica do plexo braquial (POPB) é bastante comum em neonatos, e pode se resolver de forma espontânea ${ }^{1}$ em entre 30 e 90\% dos casos. Esta lesão é causada pela tração do plexo braquial durante o trabalho de parto, e a extensão do dano nervoso só pode ser determinada pela avaliação da recuperação ao longo do tempo. ${ }^{2}$ Sua incidência é de 0,15 a 3 casos por 1.000 nascidos vivos. ${ }^{2}$ A lesão clássica é a paralisia de $\mathrm{C} 5 \mathrm{e}$ C6, mas todas as raízes nervosas podem ser acometidas. ${ }^{1}$

Enxertos de nervo são realizados na população neonatal com paralisia do plexo braquial há mais de 30 anos. Estes procedimentos são recomendados em pacientes com ruptura pós-ganglionar das raízes nervosas superiores do plexo braquial (C5 e C6). ${ }^{3}$ A cirurgia de transferência de nervo é geralmente indicada em casos de apresentação tardia, insucesso da reconstrução nervosa primária, déficit isolado, ausência de raiz proximal para enxerto e múltiplas avulsões da raiz nervosa. ${ }^{3}$ Neste procedimento, ramos de um nervo vizinho são retirados e redirecionados para a extremidade distal do nervo danificado. ${ }^{4}$ Depois da cirurgia, há regeneração dos axônios da nova via e reconexão do córtex motor para reaprendizado das funções musculares. ${ }^{4}$

Há diversos relatos experimentais nos quais o aloenxerto de nervo foi usado como alternativa ao autoenxerto para união de duas extremidades de um nervo em seres humanos e outros primatas. ${ }^{5}$ O tecido do aloenxerto atuaria como suporte temporário para maior regeneração neural, dando as características estruturais essenciais do tecido nervoso. ${ }^{6}$ O restauro da flexão do cotovelo é de grande importância, sendo uma das maiores prioridades da reconstrução do plexo braquial; ${ }^{7}$ uma das formas mais comuns para a avaliação dessa recuperação é o sistema de classificação do Medical Research Council (MRC, na sigla em inglês). ${ }^{8,9}$ Assim, a presente revisão sistemática pretende explorar o uso de enxertos e transferências de nervo como procedimentos para melhora da flexão do cotovelo em crianças com POPB.

\section{Métodos}

A presente revisão sistemática foi realizada de acordo com as diretrizes Preferred Reporting Items for Systematic Reviews and Meta-Analysis (PRISMA, na sigla em inglês) ${ }^{10} \mathrm{com}$ pesquisa nos bancos de dados MEDLINE, EMBASE, LILACS, The Cochrane Central Register of Controlled Trials (CENTRAL, sigla em inglês), Web of Science, Wholis e SCOPUS. Na busca, incluímos termos em inglês, espanhol e português usando a seguinte equação: $\mathrm{E}=($ P1 E P2 NÃO (P3 OU P4)) E I E O. A questão PICO era "quais são as evidências de melhora da flexão do cotovelo com a técnica de enxerto de nervo ou transferência de nervo em crianças com POPB?". A busca não foi restrita por tempo (Material suplementar 1).

\section{Critério de Seleção}

Os critérios pré-determinados definiram os seguintes requisitos para inclusão de um artigo: ensaios clínicos, quase-experimentos e estudos de coortes com enxertos de nervo e transferências 
de nervo em crianças ( $\leq 3$ anos de idade) com diagnóstico de POPB. Os estudos deveriam ter pelo menos 6 meses de acompanhamento de todos os desfechos. Todos os estudos comparativos de enxertos e transferências relataram desfechos de força muscular medidos pelo MRC.

\section{Extração de Dados}

Revisores independentes e cegos extraíram dados dos artigos elegíveis. As variáveis de abstração foram autor, ano do estudo, delineamento experimental, número de pacientes em cada procedimento, idade à cirurgia, gênero, lesões, período de acompanhamento e nervo doador de qualquer procedimento. $\mathrm{O}$ desfecho primário foi a análise do uso de enxertos e transferências de nervo em crianças com POPB, e o desfecho secundário foi a recuperação da flexão do cotovelo após os dois procedimentos, que foi avaliada conforme a classificação de força do MRC. Esse sistema consiste em 5 graus, em que 0 representa ausência de contração; 1 representa tremor ou traço de contração; 2 representa movimentos ativos com eliminação da gravidade; 3 representa movimentos ativos contra a gravidade; 4 representa movimentos ativos contra a gravidade e resistência; e 5 representa força normal. ${ }^{8}$ Dois pesquisadores revisaram o título e o resumo de cada estudo encontrado nos bancos de dados, escolhendo os mais adequados. Depois, revisaram os textos completos dos artigos selecionados de acordo com os critérios de inclusão. Os dados dos artigos escolhidos foram, então, extraídos. As divergências foram resolvidas por consenso e, quando a discordância não pôde ser resolvida, um dos dois revisores decidiu o conflito.

\section{Avaliação do Risco de Viés}

A ferramenta de avaliação Risk of Bias in Non-Randomized Studies of Interventions (ROBINS-I, na sigla em inglês) ${ }^{11}$ foi usada em estudos não randomizados. Esta ferramenta inclui 7 domínios específicos de viés: 1 , confusão; 2 , seleção de participantes; 3, classificação da intervenção; 4, desvio das intervenções; 5 , ausência de dados de resultados; 6, medida dos resultados; e 7 , seleção do resultado geral relatado. 0 risco de viés foi classificado como: 0 , sem informações; 1 , baixo risco; 2, risco moderado; 3 , risco grave; e 4 , risco crítico. Dois autores avaliaram de maneira independente o risco de viés dos artigos incluídos. As discordâncias foram resolvidas por consenso.

\section{Estratégia para Análise de Dados}

A análise estatística de variáveis categóricas consistiu em percentuais, frequências e medidas de tendência central.

\section{Resultados}

Nossa pesquisa bibliográfica nos diferentes bancos de dados gerou 344 registros após a remoção de duplicatas. Após a triagem de títulos e resumos, 44 estudos eram elegíveis para avaliação do texto completo. Por fim, sete estudos foram incluídos na revisão sistemática, como mostrado no fluxograma PRISMA (-Fig. 1). As discordâncias foram resolvidas por consenso

\section{Características do Estudo}

Sete estudos foram selecionados; três deles compararam os procedimentos de enxerto de nervo em 59 pacientes e transferência de nervo em 34 pacientes, com um total de 93 pacientes (-Tabela 1). Outros quatro estudos não compararam os procedimentos, mas os utilizaram como método reconstrutivo em crianças com POPB (- Tabela 1). Nos estudos que comparam o enxerto e a transferência de nervo, a idade à cirurgia variou de 5,7 a 18 meses, com período de acompanhamento de 12 a 70 meses. Nos estudos que combinaram os 2 procedimentos, a idade à cirurgia variou de 3,5 a 23 meses, com período de acompanhamento de 24,3 a 85 meses. Apenas dois dos estudos selecionados tinham todos os dados necessários para comparação do desfecho de flexão do cotovelo avaliado segundo o MRC após o enxerto ou transferência de nervo (-Tabela 2).

\section{Avaliação do Risco de Viés}

O risco de viés foi avaliado com a ferramenta ROBINS-I (Material suplementar 2). Apenas os três estudos que compararam os procedimentos de enxerto e transferência de nervo foram incluídos nesta avaliação. Nos domínios 3 e 6, 3/3 dos estudos foram classificados como baixo risco de viés; no domínio 7, 3/3 dos estudos foram classificados como risco moderado; nos domínios 2 e 4,1/3 foi classificado como risco moderado; e nos domínios 1 e 5, 1/3 foi classificado como risco grave. No geral, o risco de viés foi classificado como moderado em dois estudos e grave em um estudo.

\section{Discussão}

Nosso objetivo foi explorar o uso de enxertos e transferências de nervo como procedimentos para melhora da flexão do cotovelo em crianças com POPB. Sete estudos utilizaram os dois procedimentos; três deles compararam os procedimentos de enxerto e transferência de nervo, enquanto os outros quatro os combinaram como método reconstrutivo em crianças com POPB. De acordo com a escala do MRC, os dois métodos melhoraram igualmente a flexão do cotovelo das crianças, o que coincide com estudos anteriores. ${ }^{12}$

Chang et al. ${ }^{3}$ observaram melhora semelhante da flexão do cotovelo em abdução e adução em grupos de neonatos submetidos à transferência de Oberlin e ao enxerto de nervo, sem diferença estatística. Segundo os autores, a transferência de nervo deve ser considerada em casos de apresentação tardia, insucesso na reconstrução primária do nervo, ausência de raiz proximal para enxerto e avulsões de múltiplas raízes nervosas (lesão pré-ganglionar). Da mesma forma, Luszawski et al. ${ }^{13}$ relataram os resultados de crianças com POPB submetidas ao enxerto ou transferência de nervo. Os autores não mostram os resultados de todos os pacientes após o período de acompanhamento, mas $100 \%$ dos indivíduos submetidos à transferência de nervo (apenas um mostrado) apresentaram recuperação do músculo bíceps para grau MRC $>$ M3. Por outro lado, 77\% dos pacientes (10 de 13 mostrados) submetidos ao enxerto de nervo apresentaram recuperação do músculo bíceps para grau MRC $>$ M3. Malessy et al. ${ }^{14}$ dividiram os pacientes em grupo A e grupo B dependendo dos 

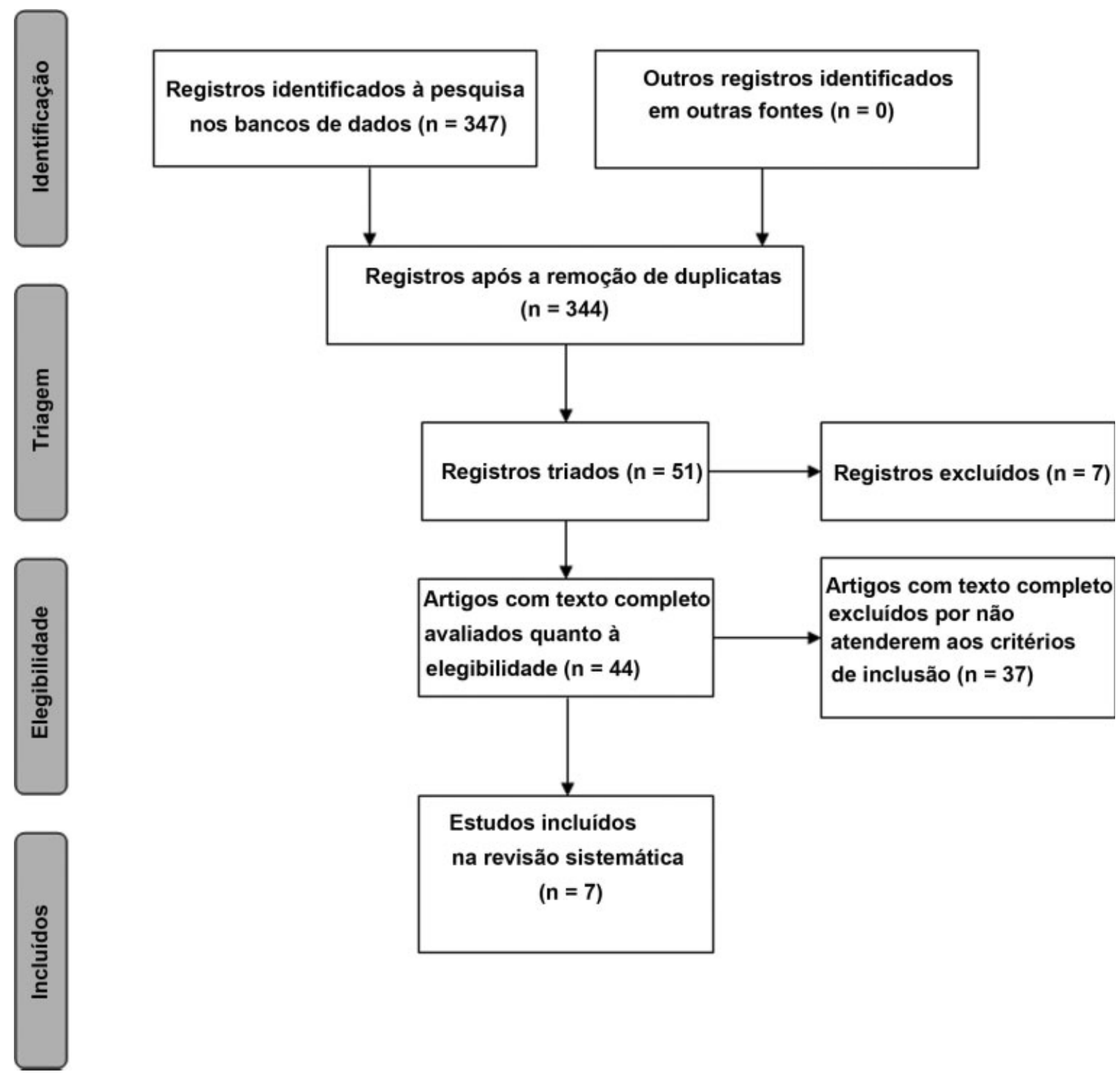

Fig. 1 Fluxograma PRISMA dos estudos escolhidos.

procedimentos realizados. No grupo A, 17 crianças foram submetidas à transferência dos filamentos da raiz anterior de $\mathrm{C} 6$ com coaptação direta em 15 indivíduos ou de todo o nervo C6 para C5. O Grupo B foi formado por 17 crianças que receberam enxertos de $\mathrm{C} 5$ para a divisão anterior do tronco superior. Segundo os autores, todos os pacientes, independentemente da cirurgia realizada, apresentaram recuperação do músculo bíceps para grau MRC $>$ M4. Outro estudo, de Yang et al., ${ }^{15}$ também comprovou a viabilidade do restauro da avulsão de C5 e C6 do plexo braquial por meio da técnica de anastomose extradural do nervo.

Bhandari et al. ${ }^{16}$ usaram neurólise e enxerto de nervo combinados à transferência de nervo como procedimentos cirúrgicos para reconstrução nervosa em crianças com POPB. A neurólise foi indicada em casos de neuroma em continuidade, ${ }^{17}$ e enxertos de nervo foram usados como pontes em defeitos nervosos, já que os neuromas não condutores foram ressectados; as transferências de nervos foram indicadas em casos de avulsões e lesões irreparáveis da raiz nervosa. ${ }^{18}$ Os pacientes com paralisia total foram submetidos à transferência e enxerto de nervo quando a estrutura a ser transferida tinha comprimento insuficiente, com 70\% de recuperação do bíceps. Os autores acreditam que há poucas indicações para neurólise na POPB, e que os resultados da ressecção do neuroma seguida de enxerto de nervo em crianças de 3 a 4 meses de idade são muito superiores. Esta afirmação foi amplamente confirmada por muitos estudos que mostram desfechos melhores das reconstruções nervosas em crianças mais novas. ${ }^{14,19}$ Além disso, Birch et al. ${ }^{20}$ não encontraram nenhuma diferença estatística no reparo de C5 por enxerto ou transferência de nervo. Terzis et $\mathrm{al}^{21}$ descobriram que, no geral, $78 \%$ dos membros submetidos à cirurgia de reconstrução do nervo apresentaram resultados bons e excelentes (M3 + ). De acordo com os autores, a reconstrução tardia ( $\sim 7$ meses) do nervo musculocutâneo teve resultados inferiores, e crianças com paralisia em C5-C6 apresentaram flexão de cotovelo com força significativamente 

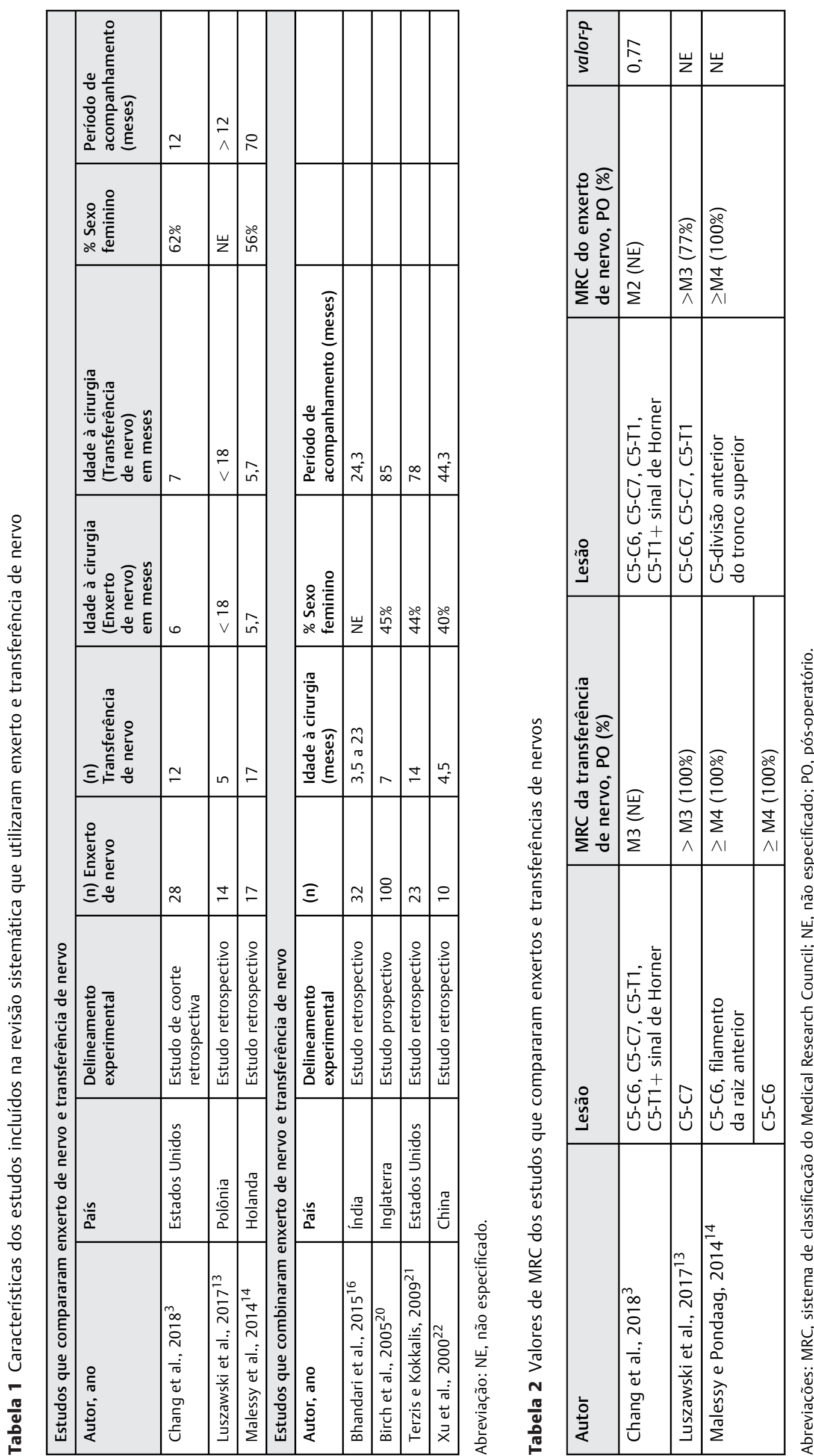
maior em comparação àquelas com paralisia global. Xu et al. ${ }^{22}$ selecionaram pacientes que não apresentaram recuperação da contração do bíceps até os 3 meses de idade. O procedimento combinado de transferência e enxerto de nervo foi realizado em 10 pacientes com POPB. Resultados excelentes e bons na flexão do cotovelo foram observados em $70 \%$ dos pacientes no grupo submetido à transferência e enxerto de nervo. Além disso, 80\% das crianças apresentaram recuperação do músculo bíceps para grau MRC $\mathrm{M} 3+$. De acordo com os autores, os resultados mostraram que a transferência combinada ao enxerto de nervo é a melhor opção para o manejo da ressecção do neuroma e a reconstrução do plexo braquial; além disso, os nervos infantis têm maior capacidade de regeneração, ${ }^{23}$ e a distância para que os axônios alcancem o órgão final é menor, ${ }^{24}$ o que melhora o resultado da cirurgia

\section{Conclusões}

No geral, nossos resultados mostraram que as duas técnicas, o enxerto de nervo e a transferência de nervo, são boas opções para reconstrução nervosa em casos de POPB. O presente estudo tem várias limitações; uma delas é que todos os artigos incluídos eram estudos não randomizados. Além disso, havia inconsistências quanto ao tipo de lesão, a abordagem cirúrgica e o tempo de acompanhamento. Mais pesquisas que abordem as técnicas de reconstrução nervosa em POPB devem ser realizadas, preferencialmente estudos clínicos randomizados, para validação dos resultados da presente revisão sistemática.

\section{Contribuições dos Autores}

Girón E. V. e Zapata-Copete J. A. contribuíram de forma substancial para a concepção, delineamento experimental, aquisição, análise e interpretação dos dados. Os dois autores participaram da redação do artigo e de sua revisão crítica quanto ao conteúdo de importância intelectual. Ambos aprovaram a versão final a ser publicada. Além disso, ambos concordaram em ser responsáveis por todos os aspectos do trabalho, assegurando que as questões relacionadas à precisão ou integridade de qualquer parte do presente artigo sejam devidamente investigadas e resolvidas.

\section{Suporte Financeiro}

Não houve suporte financeiro de fontes públicas, comerciais, ou sem fins lucrativos.

\section{Conflito de Interesses}

Os autores declaram não haver conflito de interesses a declarar.

\section{Referências}

1 Thatte MR, Mehta R. Obstetric brachial plexus injury. Indian J Plast Surg 2011;44(03):380-389

2 Pondaag W, Malessy MJ, van Dijk JG, Thomeer RT. Natural history of obstetric brachial plexus palsy: a systematic review. Dev Med Child Neurol 2004;46(02):138-144

3 Chang KWC, Wilson TJ, Popadich M, Brown SH, Chung KC, Yang LJS. Oberlin transfer compared with nerve grafting for improving early supination in neonatal brachial plexus palsy. J Neurosurg Pediatr 2018;21(02):178-184
4 Karamanos E, Rakitin I, Dream S, Siddiqui A. Nerve Transfer Surgery for Penetrating Upper Extremity Injuries. Perm J 2018;22:17-156

5 Moore AM, MacEwan M, Santosa KB, et al. Acellular nerve allografts in peripheral nerve regeneration: a comparative study. Muscle Nerve 2011;44(02):221-234

6 Chen S, Chen Z-G, Dai H, et al. Repair, protection and regeneration of peripheral nerve injury. Neural Regen Res 2015;10(11):1777-1798

7 Siqueira M, Martins R, Faglioni W Junior, Foroni L, Heise C. Restoration of Elbow Flexion in Traumatic Upper Brachial Plexus Palsy in Adults: Outcome with Intraplexus Distal Nerve Transfers in 78 Patients. Arq Bras Neurocir 2018;37(04):285-290

8 James MA. Use of the Medical Research Council muscle strength grading system in the upper extremity. J Hand Surg Am 2007;32 (02):154-156

9 Bhardwaj P, Bhardwaj N. Motor grading of elbow flexion - is Medical Research Council grading good enough? J Brachial Plex Peripher Nerve Inj 2009;4:3

10 Urrútia G, Bonfill X. [PRISMA declaration: a proposal to improve the publication of systematic reviews and meta-analyses]. Med Clin (Barc) 2010;135(11):507-511

11 Sterne JAC, Hernán MA, Reeves BC, et al. ROBINS-I: a tool for assessing risk of bias in non-randomised studies of interventions. BMJ 2016;355:i4919

12 Tora MS, Hardcastle N, Texakalidis P, Wetzel J, Chern JJ. Elbow flexion in neonatal brachial plexus palsy: a meta-analysis of graft versus transfer. Childs Nerv Syst 2019;35(06):929-935

13 Luszawski J, Marcol W, Mandera M. The components of shoulder and elbow movements as goals of primary reconstructive operation in obstetric brachial plexus lesions. Neurol Neurochir Pol 2017;51(05):366-371

14 Malessy MJA, Pondaag W. Neonatal brachial plexus palsy with neurotmesis of C5 and avulsion of C6: supraclavicular reconstruction strategies and outcome. J Bone Joint Surg Am 2014;96 (20):e174

15 Yang KX, Zhang SH, Ge DW, Sui T, Chen HT, Cao XJ. A novel extradural nerve transfer technique by coaptation of $\mathrm{C} 4$ to $\mathrm{C} 5$ and C7 to C6 for treating isolated upper trunk avulsion of the brachial plexus. J Biomed Res 2018;32(04):298-304

16 Bhandari P. Functional Outcomes of Nerve Reconstruction in Severe Obstetric Brachial Plexus Palsy. Indian J Neurotrauma 2015;12(01):41-48

17 Andrisevic E, Taniguchi M, Partington MD, Agel J, Van Heest AE. Neurolysis alone as the treatment for neuroma-in-continuity with more than 50\% conduction in infants with upper trunk brachial plexus birth palsy. J Neurosurg Pediatr 2014;13(02):229-237

18 Bhandari PS, Sadhotra LP, Bhargava P, et al. Surgical outcomes following nerve transfers in upper brachial plexus injuries. Indian J Plast Surg 2009;42(02):150-160

19 Al-Qattan MM, Al-Kharfy TM. Median nerve to biceps nerve transfer to restore elbow flexion in obstetric brachial plexus palsy. BioMed Res Int 2014;2014:854084

20 Birch R, Ahad N, Kono H, Smith S. Repair of obstetric brachial plexus palsy: results in 100 children. J Bone Joint Surg Br 2005;87 (08):1089-1095

21 Terzis JK, Kokkalis ZT. Elbow flexion after primary reconstruction in obstetric brachial plexus palsy. J Hand Surg Eur Vol 2009;34 (04):449-458

$22 \mathrm{Xu} \mathrm{J}$, Cheng X, Gu Y. Different methods and results in the treatment of obstetrical brachial plexus palsy. J Reconstr Microsurg 2000;16(06):417-420, discussion 420-422

23 Donega V, van Velthoven CT, Nijboer CH, Kavelaars A, Heijnen CJ. The endogenous regenerative capacity of the damaged newborn brain: boosting neurogenesis with mesenchymal stem cell treatment. J Cereb Blood Flow Metab 2013;33(05):625-634

24 Huelke DF. An Overview of Anatomical Considerations of Infants and Children in the Adult World of Automobile Safety Design. Annu Proc Assoc Adv Automot Med 1998;42:93-113 